\title{
Screening and identification of a Penicillium brevicompactum strain isolated from the fruiting body of Inonotus obliquus and the fermentation production of mycophenolic acid
}

\author{
Cong Min ${ }^{1} \cdot$ Hao Dong ${ }^{2} \cdot$ Xingbin Liu ${ }^{1} \cdot$ Zongshen Zhang $^{1}$ \\ Received: 28 May 2019 / Accepted: 13 September 2019/Published online: 30 October 2019 \\ (C) Università degli studi di Milano 2019
}

\begin{abstract}
Purpose Mycophenolic acid (MPA) is a fungal metabolite with a variety of biological activities and widely applied in clinical practices. We herein aimed to isolate a new Penicillium brevicompactum strain from the fruiting body of Inonotus obliquus to improve the production of MPA.

Methods The fruiting body of Inonotus obliquus was used to isolate P. brevicompactum strains. Identification of the $P$. brevicompactum strain was performed by sequencing and phylogenetic tree analysis. HPLC assay was conducted to identify the production of MPA. Submerged liquid fermentation and bi-directional fermentation were applied to improve the yield of MPA.

Result The candidates of the P. brevicompactum strain were isolated and screened from the fruiting body of Inonotus obliquus collected from Changbai mountains in China. Based on sequencing and phylogenetic tree analysis of 18S rDNA-ITS, the strain MC-4 was finally identified as P. brevicompactum. And HPLC assay indicated that the isolated P. brevicompactum strain could produce MPA in metabolic products. The optimized conditions of submerged liquid fermentation were as follows: $100 \mathrm{~g} / \mathrm{L}$ Chinese yam in a liquid PDB medium, $\mathrm{pH}$ 6, fermentation temperature of $24^{\circ} \mathrm{C}$, shaker speed of $130 \mathrm{r} / \mathrm{min}$, and fermentation time of 6 days. The maximum value of MPA production was $1.415 \mathrm{~g} / \mathrm{L}$ after submerged liquid fermentation. Furthermore, the yield of MPA could be significantly increased to $1.537 \mathrm{~g} / \mathrm{L}$ after bi-directional fermentation with the extractive from fructus Swietenia macrophylla (FSM).

Conclusion We demonstrated that a Penicillium brevicompactum strain isolated from the fruiting body of Inonotus obliquus can be used to improve the production of MPA by submerged liquid fermentation and bi-directional fermentation. This would provide a novel approach for more efficient and safer production of MPA.
\end{abstract}

Keywords Mycophenolic acid, Inonotus obliquus, P. brevicompactum, Fructus Swietenia macrophylla, Bi-directional fermentation

\section{Introduction}

Mycophenolic acid (MPA) is a fungal metabolite that mainly exists in the Penicillium genus, including $P$. brevicompactum, $P$. stoloniferum, $P$. viridicatum, and

Zongshen Zhang

zhangzs@dlpu.edu.cn

1 School of Biological Engineering, Dalian Polytechnic University, Qinggongyuan No.1, Ganjingzi District, Dalian 116034, China

2 The College of Life Sciences, Northwest University, Xi'an 710069, China others (Regueira et al. 2011). MPA was firstly discovered in 1893 and has been identified as an important bioactive substance with immunosuppressive, antiviral, antitumor, antibacterial, antifungal, and various activities (Carter et al. 1969; Hood and Zarembski 1997; Vinci et al. 2018). Notably, its derivative, mycophenolate mofetil, is a key immunosuppressant that is widely applied in the clinical transplantation of the liver, kidney, and heart (Okour et al. 2018; Zhang et al. 2018).

Because of the excellent medicinal value of MPA, optimization for MPA production process has always been a research hotspot. Currently, MPA is mainly produced by fungal fermentation, including solid state and 
liquid state fermentation (Xu and Yang 2007). However, traditional fermentation has a great limitation in MPA production due to the low efficiency and productivity of fungal strain, the difference in fermentation approach, and the deficiency of technological parameter. Most importantly, there are often toxic and side effects in MPA produced by traditional fermentation, which restrict the use of MPA in clinical practice (Mudge et al. 2004). Hence, further exploring new fungal strain and fermentation method with higher efficiency is necessary for the application of MPA.

Penicillium brevicompactum, one of the main members in the Penicillium genus, is a kind of filamentous fungus with highly medicinal value. As the main fungal strain producing MPA, $P$. brevicompactum is widely used in fermentation production of MPA (Regueira et al. 2011; Xu et al. 2017). P. brevicompactum is distributed throughout the natural world and can be isolated from soil, rotting vegetables, food, grains, textiles, dyes, and various other sources (Xu et al. 2017). Nevertheless, different $P$. brevicompactum strains derived from diverse sources often have varying efficiency in fermentation production along with different yield of MPA.

Inonotus obliquus, also known as Chaga, is a parasitic fungus that has broad-spectrum health benefit in traditional medicine for various health problems (Cui et al. 2005; Nakajima et al. 2007). As a kind of medicinal and edible fungus, Inonotus obliquus is generally parasitic on the birch trees with many years old. In the long-term parasitic processes of Inonotus obliquus, there often are some other beneficial microorganisms that may also live symbiotically or parasitically with the fruiting body of Inonotus obliquus, to survive by using the nutrition of birch tree or Inonotus obliquus (Géry et al. 2018; Wold et al. 2018). In the present study, we isolated and identified a P. brevicompactum strain from the fruiting body of Inonotus obliquus. Furthermore, we applied the P. brevicompactum strain isolated from Inonotus obliquus to improve the production of MPA by submerged liquid fermentation and bi-directional fermentation with the extractive from fructus Swietenia macrophylla (FSM). Our results would provide a novel approach for more efficient and safer production of MPA.

\section{Materials and methods}

\section{Materials}

The wild fruiting body of Inonotus obliquus was collected from birch with many years old in Changbai mountains in Jilin (China). FSM was purchased from Solomon Islands by online purchasing. Chinese yam was purchased from Wenxian (Henan, China) by online purchasing. The standard of MPA was purchased from Zhongkezhijian Biotechnology Co. Ltd (Beijing, China).

\section{Screening of $P$. brevicompactum strain}

Nine cubes $\left(0.5 \mathrm{~cm}^{3}\right)$ of the fruiting body of Inonotus obliquus were obtained using a knife from three different parts in the fruiting body of Inonotus obliquus. Each cube of the fruiting body of Inonotus obliquus was washed with $1 \mathrm{~mL}$ sterile water for $4-5$ times. The washing fluid was collected in a sterile condition and planted on the PDA solid medium (200 g potato, $18 \mathrm{~g}$ agar, $20 \mathrm{~g}$ glucose $/ 1 \mathrm{~L}$ medium, $\mathrm{pH}=6.0$ ) containing cephalosporin $\mathrm{C}(10 \mu \mathrm{g} / \mathrm{mL})$ for $6-12$ days at $28{ }^{\circ} \mathrm{C}$. Then, the colony was collected and planted on the PDA solid medium containing cephalosporin $\mathrm{C}$ to culture at $28{ }^{\circ} \mathrm{C}$. After $6-12$ days, the above steps were repeated until a purified single colony was obtained.

\section{Identification of $P$. brevicompactum strain}

The purified single colony was cultured in the PDA liquid medium containing cephalosporin $\mathrm{C}$ for $48 \mathrm{~h}$ at $24{ }^{\circ} \mathrm{C}$ in a shaking table at $130 \mathrm{r} / \mathrm{min}$. After standing for $24 \mathrm{~h}$, pale yellow mycelium was collected by filtration and washing. The genomic DNA was extracted from mycelium strain through a benzyl chloride method (Yang et al. 2014). PCR assay was then performed to obtain the ITS sequences of fungi strain. The primers were used as follows: ITS1: 5'-TCCGTAGGTGAACC TGCGG-3', and ITS4: 5'-TCCTCCGCTTATTG ATATGC-3'. PCR fragments were then conducted with sequencing identification. After a manual correction with BioEdit v.7.0, sequence alignment (BLAST) was performed with homologous $P$. brevicompactum for strain identification in public databases (http://www. ncbi.nlm.nih.gov/BLAST/).

And phylogenetic tree was constructed by MEGA 6.0 using the N-J method according to BLAST results. The fungal colony was preserved in a refrigerator at $4{ }^{\circ} \mathrm{C}$ for short-term preservation and in liquid nitrogen for long-term storage.

\section{HPLC analysis of MPA}

Fermentation products were filtrated with gauze and centrifuged at $3 \times 10^{3} \mathrm{r} / \mathrm{min}$ for $5 \mathrm{~min}$, followed by filtration by a $0.45-\mu \mathrm{m}$ filter. Extracted solution was analyzed by 
HPLC assay using Agilent 1260 Series LC system. Related parameters were as follows: Phenomenex ${ }^{\circledR}$ Luna 5 u C18 column $(250 \mathrm{~nm} \times 4.6 \mathrm{~mm})$, mobile phase (phosphoric acid/water 40:60), detecting wavelength $240 \mathrm{~nm}$, flow rate $1 \mathrm{~mL} / \mathrm{min}$, and sample amount $20 \mu \mathrm{L}$. Sample was identified by comparing the retention time and spectra with MPA standard.

\section{Submerged liquid fermentation of $P$. brevicompactum}

The effects of Chinese yam content, speed of shaking table, initial $\mathrm{pH}$, and temperature on the production of MPA were studied. Peeled and cleaned Chinese yam was sliced and homogenized, then different concentrations of Chinese yam were added into the medium, followed by high temperature and high pressure sterilization. For culture of $P$. brevicompactum, the collected $P$. brevicompactum strain was firstly seeded on the PDA solid medium containing cephalosporin $\mathrm{C}$ for 6 days at $28{ }^{\circ} \mathrm{C}$. Then, $P$. brevicompactum was collected using inoculating loop $\left(0.5 \mathrm{~cm}^{2}\right)$ from $P$. brevicompactum colonies with the same thickness on medium. And $P$. brevicompactum from one inoculating loop was inoculated in a $100-\mathrm{mL}$ medium with $\mathrm{pH} 6$ in a conical flask, conducted with liquid fermentation at $24{ }^{\circ} \mathrm{C}$ in a shaking table at $130 \mathrm{r} / \mathrm{min}$. Three repeats were conducted for each group. The production of MPA was analyzed by HPLC analysis at 2, 4, 6, 8, and 10 days after fermentation.

\section{Bi-directional fermentation for MPA production}

Bi-directional fermentation for MPA production was performed by incubating with the extractive from FSM. In brief, FSM extractive was prepared by an ultrasonic method as the following parameters: methanol $85 \%$, extraction ratio $1: 15$, time $40 \mathrm{~min}$, and temperature $70{ }^{\circ} \mathrm{C}$. Then, different concentrations $(0,0.1,0.2$, $0.3,0.4,0.5 \mathrm{~g} / \mathrm{L})$ of FSM extractive were added into the 100-mL liquid PDB medium (200 g potato, $20 \mathrm{~g}$ glucose/1 L medium, $\mathrm{pH}=6.0$ ) supplemented with $100 \mathrm{~g} / \mathrm{L}$ Chinese yam. The $P$. brevicompactum strain was seeded in the above medium and incubated for 6 days at $24{ }^{\circ} \mathrm{C}$ in a shaking table at $130 \mathrm{r} / \mathrm{min}$. The production of MPA was analyzed by HPLC analysis.

\section{Statistical analysis}

Data were expressed as mean \pm SEM. Differences between groups were analyzed by one-way ANOVA using GraphPad Prism 6 (La Jolla, CA, USA). All experiments were performed independently at least three times. Statistical significance was defined by $P<0.05$.

\section{Results}

\section{Screening and characteristics of $P$. brevicompactum strain}

To improve the production of MPA, we firstly isolated a $P$. brevicompactum strain from the fruiting body of Inonotus obliquus. As shown in Fig. 1, we screened potential $P$. brevicompactum strains by single colony and antibiotic screening method. Five possible strains were isolated from the fruiting body of Inonotus obliquus collected from Changbai mountains in China. The colonial morphology was seen in Fig. 2. Among all strains, MC-4 gave the characteristics of the $P$. brevicompactum strain that colony grew densely with villous texture, spore-producing structure was formed in large quantities, it was livid on the spore surface and light brown on the underside of colony, and there was no water-soluble pigment. Therefore, MC-4 was primary selected as the candidate of the $P$. brevicompactum strain.

\section{Sequencing identification of $P$. brevicompactum strain}

We further performed the sequencing analysis by $18 \mathrm{~S}$ rDNA-ITS assay to identify the $P$. brevicompactum strain from five possible strains. The genomic DNA extracted from five strains were shown in Fig. 3a. Then, PCR assay was conducted with the primers of ITS1/ITS4. There were amplification products with $600 \mathrm{bp}$ in all five strains. And sequence similarities approached $99 \%$ with the candidate sequences at GenBank in all cases, as seen in Table 1. According to the BLAST, strain MC-1 was identified as Penicillium rudallense, MC-2 and MC-3 were identified as Alternariaster helianth, MC-4 was identified as Penincillium brevicompactum, and MC-5 was Cladosporium sp. HNC12-38. Then, we constructed the phylogenetic tree of strain MC-4 based on ITS sequences. The strain MC-4 had the highest homology with $P$. brevicompactum (Fig. 3b, c). Taken together, these results suggest that the isolated strain MC-4 is P. brevicompactum and was selected for further study. 

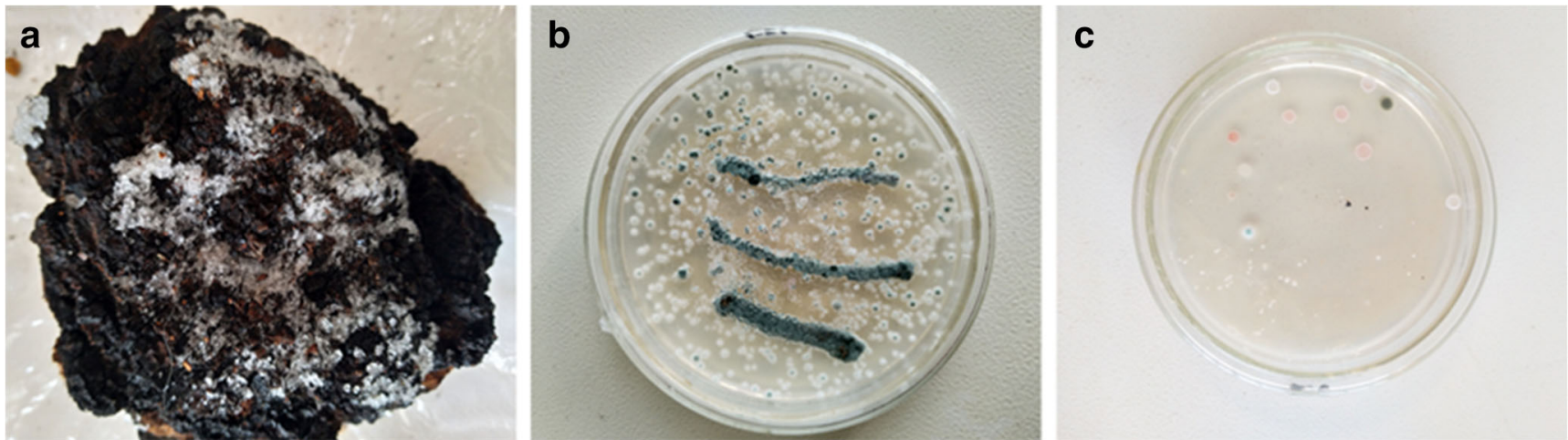

Fig. 1 Screening of the P. brevicompactum strain. The cubes of the fruiting body of Inonotus obliquus were collected and cultured on the PDA solid medium containing cephalosporin C $(10 \mu \mathrm{g} / \mathrm{mL})$ for $6-$ 12 days at $28{ }^{\circ} \mathrm{C}$. Then, the colony was collected and planted on the
PDA solid medium at same condition. The above steps were repeated until a purified single colony was obtained. a The primary fruiting body of Inonotus obliquus. b The screening of strains. c Cloning and purification of single colony

\section{HPLC identification for the fermentation product of $P$. brevicompactum}

To further determine the feasibility of isolated $P$. brevicompactum as the candidate strain for production of MPA, we analyzed the fermentation products of strain MC-4 by HPLC assay. Firstly, we confirmed that the parameters of HPLC assay for MPA standard were as follows: C18 column $(250 \mathrm{~nm} \times 4.6 \mathrm{~mm}, 5 \mu \mathrm{m})$, mobile phase $(0.1 \%$ phosphoric acid/water 40:60), detecting wavelength $240 \mathrm{~nm}$, flow rate $1 \mathrm{~mL} / \mathrm{min}$, temperature $40{ }^{\circ} \mathrm{C}$, and sample amount $20 \mu \mathrm{L}$ (Fig. 4a). Compared with the MPA standard, there was a spectrum peak in the HPLC result of liquid fermentation broth from strain MC-4 at the same retention time 4.763 min with MPA standard (Fig. 4b). And the concentration of MPA was $65.40 \%$ in the sample of fermentation broth. This data indicate that the isolated $P$. brevicompactum strain can produce MPA in metabolic products.

\section{Submerged liquid fermentation to improve the yield of MPA}

As submerged liquid fermentation is an efficient method for obtaining fermentation products, we herein conducted and
Fig. 2 Characteristics of P. brevicompactum strain candidates. Five single colonies were selected as the candidates of P. brevicompactum strain. The characteristics of MC-1, MC-2, MC-3, MC-4, and MC-5

candidate strains were showed
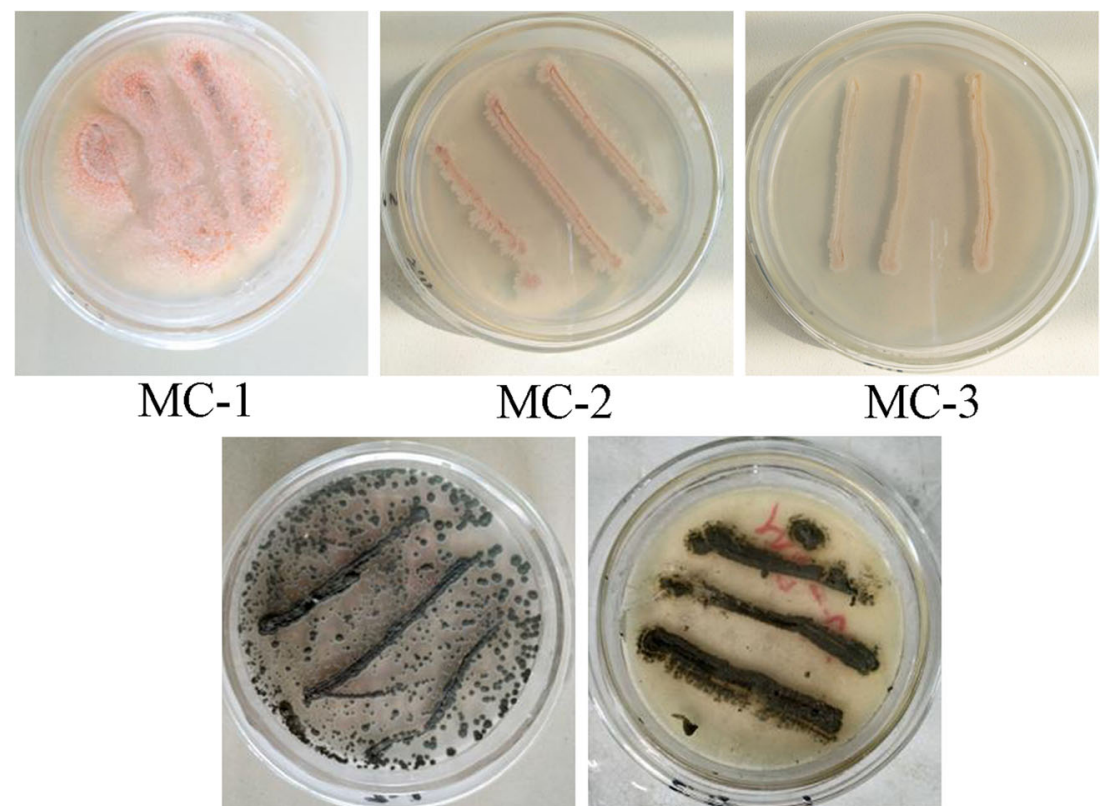

$\mathrm{MC}-4$

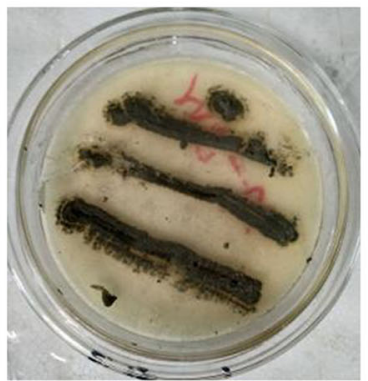

$\mathrm{MC}-5$ 


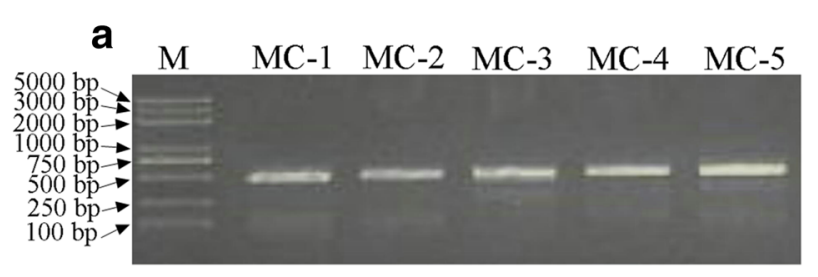

b

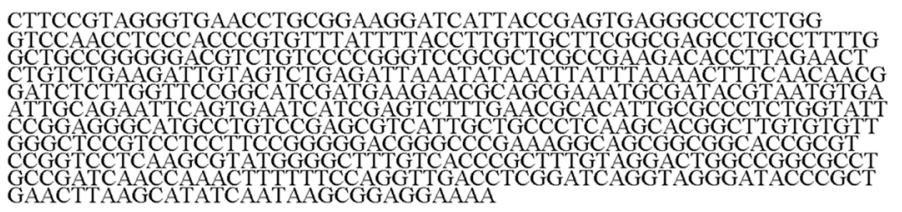

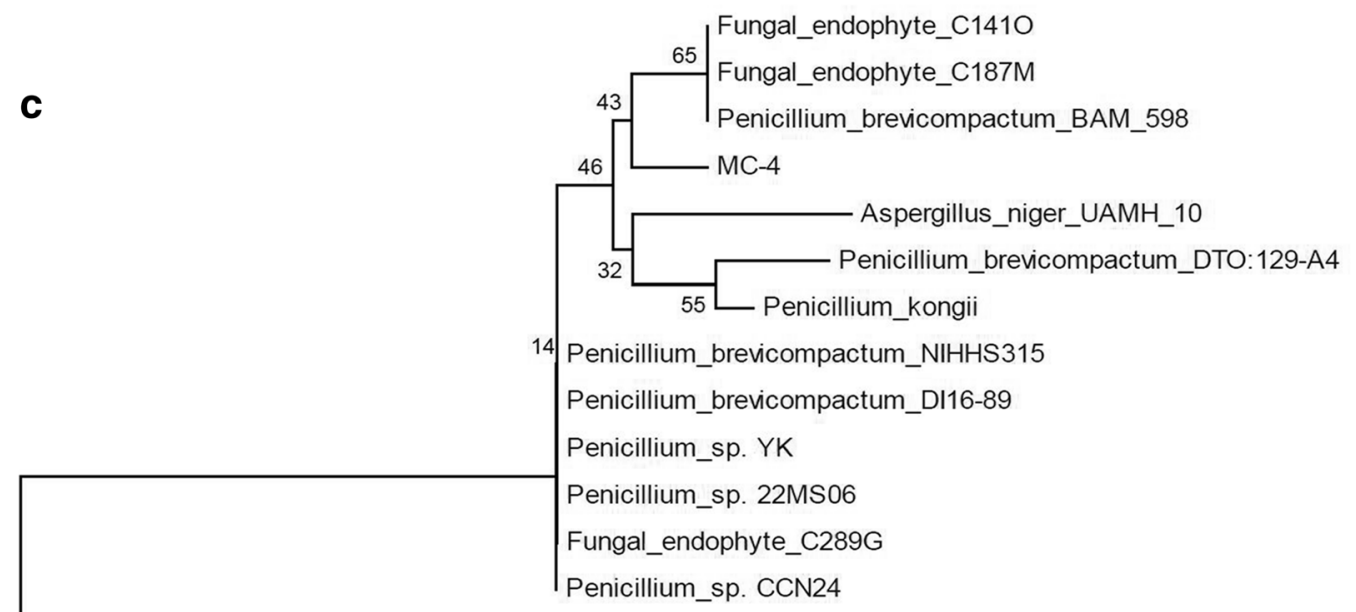

Fungal_sp. clone_PdIM07-12

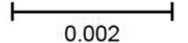

Fig. 3 Sequencing identification of the $P$. brevicompactum strain. a The genomic DNA was extracted from five candidate strains and detected by electrophoresis assay. Then, PCR assay was conducted with the primers of ITS1/ITS4. b The sequence of candidate strain MC-4 was showed. $\mathbf{c}$ The phylogenetic tree of strain MC-4 was constructed based on the ITS sequences by the N-J method optimized the submerged liquid fermentation of the isolated $P$. brevicompactum strain to improve the yield of MPA. Firstly, the effects of Chinese yam content, initial $\mathrm{pH}$, temperature, and speed of shaking on the production of MPA were investigated (Fig. 5A-D). We finally performed the submerged liquid fermentation of strain $\mathrm{MC}-4$ in the liquid PDB medium supplemented with $100 \mathrm{~g} / \mathrm{L}$ Chinese yam as source of nutrients at $24^{\circ} \mathrm{C}$ and $\mathrm{pH} 6$ condition in a shaking table at $130 \mathrm{r} / \mathrm{min}$. Besides, we determined the effect of fermentation time on the production of MPA. Results showed that the yield of MPA was increased over time and reached the peak at 6 days after fermentation (Fig. 5E).

Furthermore, there was a significant change in the morphological feature of mycelium pellets of strain MC-4. The mycelium pellets have comparatively smaller volume with irregular spherical shape in the primary fermentation, whereas the volume of mycelium pellets were obviously enhanced and the shape were changed to regular sphere after submerged liquid fermentation (Fig. 5F). The maximum value of MPA production was $1.415 \mathrm{~g} / \mathrm{L}$ after submerged liquid fermentation, which was significantly higher than $0.856 \mathrm{~g} / \mathrm{L}$ in primary fermentation. These results indicate that the production of
MPA from the isolated $P$. brevicompactum strain MC-4 could be effectively improved by submerged liquid fermentation.

\section{Bi-directional fermentation to improve the properties of MPA}

Bi-directional fermentation is a new Chinese herbal fermentation technology that can effectively reduce the toxicity and improve the production of fermentation products (Xia and Pan 2010). In the present study, we performed the bi-directional fermentation of isolated $P$. brevicompactum strain with the extractive from FSM to further improve fermentation production of MPA. Firstly, FSM extractive was prepared by an ultrasonic method with the yield of $2.189 \mathrm{mg}$ alcohol soluble substance in $1 \mathrm{~g}$ FSM. Then, the FSM extractive was added into the fermentation broth of $P$. brevicompactum, followed by submerged liquid fermentation. As seen in Fig. 6 a and b, yield of MPA was gradually enhanced with the increase of FSM extractive concentration and reached the peak $(1.537 \mathrm{~g} / \mathrm{L})$ when the concentration of FSM extractive was $3 \mathrm{~g} / \mathrm{L}$. And there were also changes in the phenotypic characteristic of fermentation products. Taken together, these 
Table 1 The identification of five Inonotus obliquus-derived candidate strains through sequencing of ITS1/ITS4 gene

\begin{tabular}{lllll}
\hline Strains & Closest relative species & Accession number & Total scores & Identity percentage \\
\hline MC-1 & Penicillium rudallense & LT558912.1 & 1066 & 99 \\
MC-2 & Alternariaster helianth & AY154713.1 & 1026 & 99 \\
MC-3 & Alternariaster helianth & AY154713.1 & 1038 & 99 \\
MC-4 & Penincillium brevicompactum & LT558911.1 & 1075 & 99 \\
MC-5 & Cladosporium sp. HNC12-38 & KT989383.1 & 941 & 99 \\
\hline
\end{tabular}

results suggest that the Penicillium brevicompactum strain isolated from the fruiting body of Inonotus obliquus can be used to further improve the production of MPA by bi-directional fermentation with FSM extractive on the basis of submerged liquid fermentation.

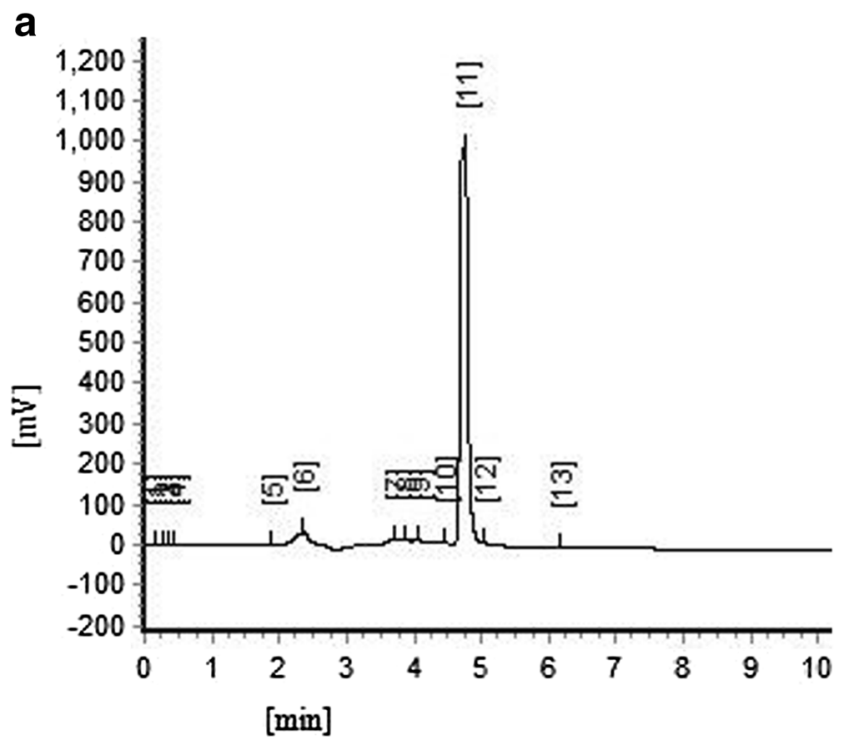

b

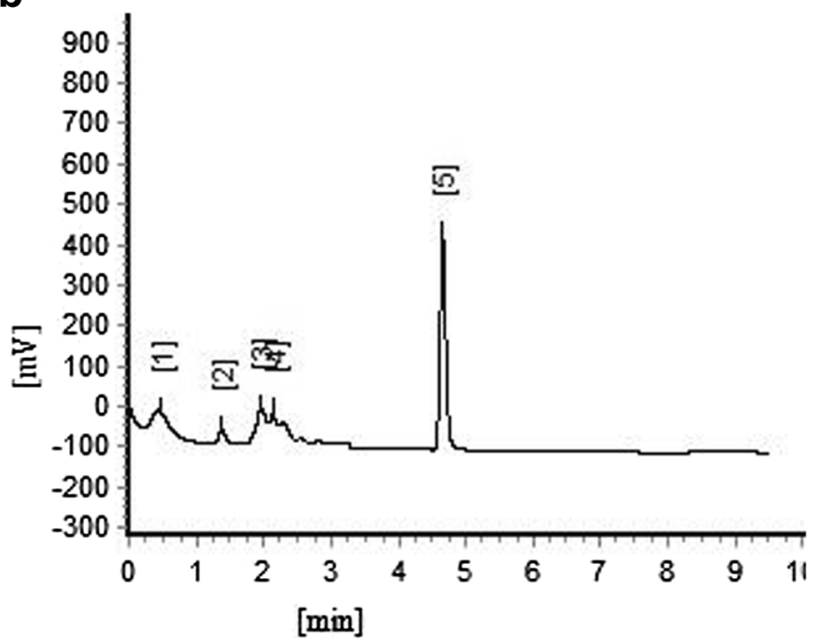

Fig. 4 HPLC identification for the fermentation product of the P. brevicompactum strain MC-4. a The HPLC analysis of MPA standard. b The HPLC result of liquid fermentation broth from the P. brevicompactum strain MC-4

\section{Discussion}

Mycophenolic acid (MPA) is a crucial fungal metabolite that has immunosuppressive, antiviral, antitumor, antibacterial, antifungal, and various bioactivities, and widely applied in clinical practice (Juki et al. 2006; Domhan et al. 2008; Morales et al. 2008; Heischmann et al. 2017). Therefore, it is always the research hotspot that finding more efficient strain producing MPA and optimizing the production process of MPA. In the present study, a multiphasic approach was applied to obtain the excellent P. brevicompactum strain and to improve the fermentation production of MPA.

Previous reports have demonstrated that screening on strain sources is necessary to obtain the suitable culture strains for fermentation production of MPA because of the greatly intraspecific differences (Vinokurova et al. 2005; Xu and Yang 2007). The main sources of MPA are the Penicillium genus, such as $P$. brevicompactum and $P$. viridicatum, which are widely distributed in food and other resources (Shaligram et al. 2008; Dong et al. 2015; Xu et al. 2017). Inonotus obliquus, an edible and medicinal fungus, is applied in food and health products in traditional medicine (Cui et al. 2005; Frisvad et al. 2013; Géry et al. 2018). And Inonotus obliquus has also been used to produce polysaccharides, phenols, and other secondary metabolites by specific liquid fermentation (Baek et al. 2018). However, it has not been used as the source of strain producing MPA in previous studies. We herein firstly isolated and identified a $P$. brevicompactum strain MC-4 producing MPA from the fruiting body of Inonotus obliquus by single colony screening and sequencing identification.

Notably, we also screened other four fungal strains from the fruiting body of Inonotus obliquus that belongs to the Penicillium genus and other imperfect fungi. A possible reason for this might be associated with the unknown complex composition of Inonotus obliquus as a parasitic fungus. Inonotus obliquus is generally parasitic on the birch trees with many years old and takes a long time to grow, in which there are some other microorganisms that may also live symbiotically or parasitically with the fruiting body of Inonotus obliquus (Géry et al. 2018; Wold et al. 2018).

Compared to traditional fermentation, submerged liquid fermentation has many advantages of shorter fermentation cycle, larger fermentation scale, and higher fermentation yield. 

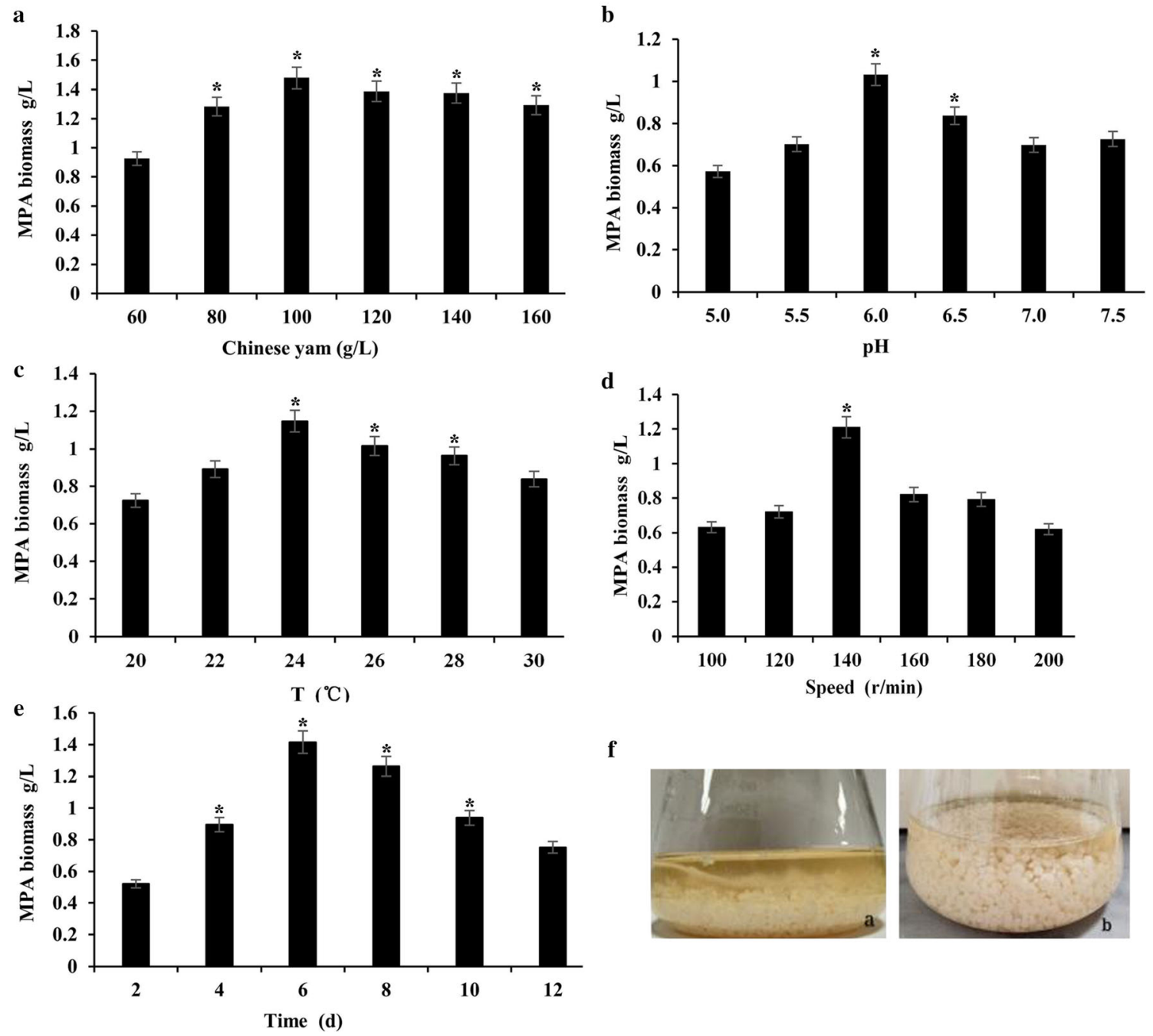

f

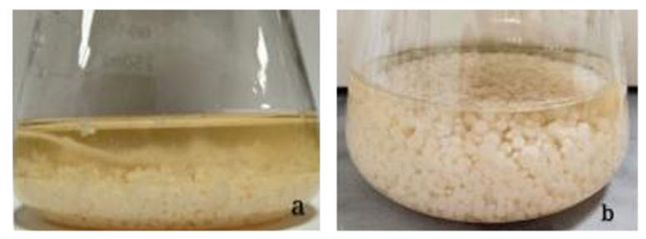

Fig. 5 Submerged liquid fermentation of the P. brevicompactum strain MC-4. The collected P. brevicompactum strain MC-4 was seeded in a $100-\mathrm{mL}$ medium with different Chinese yam content and $\mathrm{pH}$ conditions in a conical flask, conducted with liquid fermentation at different temperatures in a shaking under different speed, respectively. Then, AD effects of Chinese yam content, $\mathrm{pH}$, fermentation temperature, and

Additionally, it can increase the quantity of mycelium from fungal strain and produce more polysaccharides, terpenoids, vitamins, and other active ingredients, contributing to improve the efficacy, security, and quality control of fermentation products as potential drugs (Fazenda et al. 2008; Chi et al. 2018). In this study, we performed the submerged liquid fermentation with the isolated Inonotus obliquus-derived P. brevicompactum strain MC-4 to improve the production of MPA. The maximum value of MPA product was significantly increased to $1.415 \mathrm{~g} / \mathrm{L}$ after submerged liquid fermentation, which was significantly higher than those in primary fermentation.

There are various factors that can influence the production of submerged liquid fermentation, including sugar content, speed of shaking table, initial $\mathrm{pH}$, temperature, and fermentation time (Huang et al. 2009; Meng et al. 2016). We employed shaking speed on the production of MPA were tested. $\mathbf{E}$ The production of MPA was analyzed at 2, 4, 6, 8, and 10 days after fermentation. F The changes in the morphological feature of mycelium pellets of the $P$. brevicompactum strain MC-4 were analyzed in traditional (a) and (b) submerged liquid fermentation $(* P<0.05$ vs. $60,5.0,20,100,2)$

the Chinese yam that contains abundant sugar, protein, vitamin, free amino acids, and variety of trace elements, to provide polysaccharides and nutrients for submerged liquid fermentation of the P. brevicompactum strain (Kong et al. 2009). Basing on the metabolism and life activities of P. brevicompactum, active components in Chinese yam can be bio-transformed, furthering to improve the metabolites and production of $P$. brevicompactum in submerged liquid fermentation (Dong et al. 2015; Xu et al. 2017). Herein, we observed significant increase of MPA production in submerged liquid fermentation with Chinese yam compared to those in traditional fermentation, that is consistent with previous reports in which Chinese yam was also used in fermentation to improve the properties of production (Kong et al. 2009; Shin et al. 2006). 
a

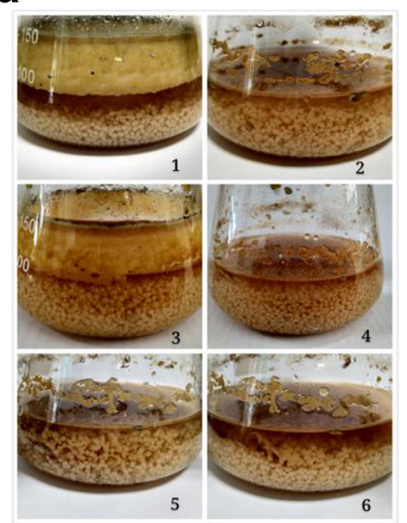

Fig. 6 Bi-directional fermentation of the P. brevicompactum strain MC-4. The FSM extractive was prepared from fructus Swietenia macrophylla by the ultrasonic method and was added into the fermentation broth of P. brevicompactum, followed by submerged liquid fermentation. a The

Besides, temperature and/or $\mathrm{pH}$ could affect the activity of enzymes in fungal strains, to influence the production of fermentation substances. And the shaking speed can change fermentation quality by affecting the formation of mycelium pellets (Shih et al. 2009; Jagadeeshbabu and Viswanathan 2010). We determined the optimal condition of submerged liquid fermentation as temperature $24{ }^{\circ} \mathrm{C}, \mathrm{pH} 6$, and shaking speed at $130 \mathrm{r} / \mathrm{min}$. Another factor affecting the yield of fermentation products is fermentation time (Wang et al. 2016). We observed that yield of MPA products reached the peak at 6 days after submerged liquid fermentation. This might be associated with the changes of mycelium pellets and biomass accompanied with the change of fermentation time. In submerged liquid fermentation, the $P$. brevicompactum strain goes into adjustment period at $0-2$ days, logarithmic growth period at 26 days, stable growth period at $6-8$ days, and decline period at $8-12$ days.

Bi-directional fermentation is a new modern fermentation technology by constituting the mixed fermentation with medical fungi and traditional Chinese medicines (Yi et al. 2007). Through bi-directional fermentation, medical fungi can obviously improve the production and properties of fermentation products compared to those in traditional fermentation. And the toxic components of Chinese medicines, including alkaloid, lactone substances, glycoside compounds, toxic protein, heavy metals, and aristolochic acids anthraquinone and tannin, can be significantly reduced (Xia and Pan 2010; Liu et al. 2018). In previous literature reports, bi-directional fermentation technology has been widely used in fermentation applications related to food, medicine, cosmetics, and other industries and achieved very good application effects (Liang 2017; Ming et al. 2010; Chong et al. 2018).

Fructus Swietenia macrophylla (FSM), also known as Swietenia macrophylla King, is the fruit of a Meliaceae plant growing in Solomon Islands. There are abundant compounds in the fruit of FSM, such as saponins, flavonoids, proteins, vitamins, and minerals, which contribute to the clinical application of FSM in treating diabetes, hyperlipidemia, hypertension, and other chronic non-communicable diseases (Lowe et al. 2003; Dewanjee et al. 2009). However, the negative factors, including bitter and side effect toxicity, limit the medicinal value of FSM. And there is cytotoxicity in the P. brevicompactum strain, which affect the production and application of MPA and other metabolites (Frisvad et al. 2013; Géry et al. 2018). Hence, based on the characteristics of $P$. brevicompactum and FSM, we performed the bi-directional fermentation of the isolated $P$. brevicompactum strain with FSM extractive for the first time. The yield of MPA was remarkably increased to $1.537 \mathrm{~g} / \mathrm{L}$ compared to those in submerged liquid fermentation, with changed phenotypic characteristics of MPA products. This is mainly associated with the advantages of bi-directional fermentation technology (Ming et al. 2010; Chong et al. 2018; Sun et al. 2018). In the specific bi-directional fermentation, medicinal fungus $P$. brevicompactum was used as the fermentation strain, to form a fermentation combination with the medicinal matrix from FSM extractive. On the one hand, the active ingredients from FSM extractive can neutralize and improve the cytotoxicity of $P$. brevicompactum, thus increasing the production of MPA (Djalil et al. 2018; Dharmalingam et al. 2012). On the other hand, metabolites and fermentation of $P$. brevicompactum can also neutralize the side effect toxicity of FSM, achieving mutual benefits (Shaligram et al. 2008).

However, our study is not without its limitation. Whether and what changes are there in the properties of MPA products after bi-directional fermentation with FSM extractive should be determined via further pharmacological experiments in the future study. 


\section{Conclusion}

We demonstrated that a Penicillium brevicompactum strain isolated from the fruiting body of Inonotus obliquus can be used to improve the production of MPA by bi-directional fermentation with FSM extractive on the basis of submerged liquid fermentation. These findings would provide a novel approach for more efficient and safer production of MPA.

Funding information This study was funded by The Excellent Talents Fund Project of Henan (No.134200510002).

\section{Compliance with ethical standards}

Conflict of interest The authors declare that they have no conflict of interest.

\section{Research involving human participants and/or animals N/A}

Informed consent N/A

\section{References}

Baek J, Roh HS, Baek KH, Lee S, Lee S, Song SS, Kim KH (2018) Bioactivity-based analysis and chemical characterization of cytotoxic constituents from Chaga mushroom (Inonotus obliquus) that induce apoptosis in human lung adenocarcinoma cells. J Ethnopharmacol 224:63-75

Carter SB, Franklin TJ, Jones DF, Leonard BJ, Mills SD, Turner RW, Turner WB (1969) Mycophenolic acid: an anti-cancer compound with unusual properties. Nature 223(5208):848-850

Chi Y, Qiao L, Zuo S, Yan H, Du C, Hwang H, Peng W (2018) Submerged liquid fermentation of Ulva prolifera Müller tissue and applications for improving growth performance in juvenile abalone (Haliotis discus hannai Ino). J Appl Phycol 30:2639-2647

Chong Z, Fang Y, Qing Q (2018) Establishment of bidirectional fermentation system of Paecilomyces cicadae/Astragalus membranaceus and study on its components. World Chinese Medic 12:44-50

Cui Y, Kim D-S, Park K-C (2005) Antioxidant effect of Inonotus obliquus. J Ethnopharmacol 96(1-2):79-85

Dewanjee S, Maiti A, Das AK, Mandal SC, Dey SP (2009) Swietenine: a potential oral hypoglycemic from Swietenia macrophylla seed. Fitoterapia 80(4):249-251

Dharmalingam K , Tan BK , Mahmud MZ (2012) Swietenia macrophylla extract promotes the ability of Caenorhabditis elegans to survive Pseudomonas aeruginosa infection. J Ethnopharmacol 139(2):0-663

Djalil AD, Genatrika E, Utaminingrum W (2018) Antidiabetic effect of mahogany (Swietenia macrophylla King) leaves extracts in glucose induced diabetic rats. Adv Sci Lett 24(1):100-102

Domhan S, Muschal S, Schwager C, Morath C, Wirkner U, Ansorge W, Maercker C, Zeier M, Huber PE, Abdollahi A (2008) Molecular mechanisms of the antiangiogenic and antitumor effects of mycophenolic acid. Mol Cancer Ther 7(6):1656-1668

Dong Y, Rui X, Wang L, Jian Z, Bai C, Sun A, Wei D (2015) A combined feeding strategy for enhancing mycophenolic acid production by fed-batch fermentation in Penicillium brevicompactum. Process Biochem 50(3):336-340

Fazenda ML, Seviour R, Mcneil B, Harvey LM (2008) Submerged culture fermentation of "higher fungi": the macrofungi. Adv Appl Microbiol 63:33-103
Frisvad JC, Houbraken J, Popma S, Samson RA (2013) Two new Penicillium species Penicillium buchwaldii and Penicillium spathulatum, producing the anticancer compound asperphenamate. FEMS Microbiol Lett 339(2):77-92

Géry A, Dubreule C, André V, Rioult JP, Bouchart V, Heutte N, Eldin dPP, Krivomaz T, Garon D (2018) Chaga (Inonotus obliquus), a future potential medicinal fungus in oncology? A chemical study and a comparison of the cytotoxicity against human lung adenocarcinoma cells (A549) and human bronchial epithelial cells (BEAS2B). Integr Cancer Ther 17:832-843

Heischmann S, Dzieciatkowska M, Hansen K, Leibfritz D, Christians U (2017) The immunosuppressant mycophenolic acid alters nucleotide and lipid metabolism in an intestinal cell model. Sci Rep 7(1):45088. https://doi.org/10.1038/srep45088

Hood KA, Zarembski DG (1997) Mycophenolate mofetil: a unique immunosuppressive agent. Am J Health Syst Pharm 54(3):285-294

Huang QY, Lin WF, Chen Z (2009) Optimization of submerged fermentation conditions for longan vinegar production. Modern Food Sci Technol 12:1419-1422

Jagadeeshbabu PE, Viswanathan R (2010) Studies on the effect of pH, temperature and metal ions on the production of pectinase from tamarind kernel powder by submerged fermentation using Aspergillus foetidus (NCIM 505). Asia-Pacific J Chemic Eng 5(2): 396-400

Juki N, John R, Juliet B, Daniel W, Jacobson PA (2006) A limited sampling model for estimation of total and unbound mycophenolic acid (MPA) area under the curve (AUC) in hematopoietic cell transplantation (HCT). Ther Drug Monit 28(3):394-401

Kong XF, Zhang YZ, Wu X (2009) Fermentation characterization of Chinese yam polysaccharide and its effects on the gut microbiota of rats. Inter J Micro 2009:1-13

Liang B (2017) Optimization of the Ganoderma lucidum-Ginkgo biloba bi-directional liquid fermentation condition and antioxidation properties of its products. Mycosystema 10:31-37

Liu Z, Tang Y, Zhou R, Shi X, Zhang H, Liu T, Lian Z, Shi X (2018) Bidirectional solid fermentation products of Trametes robiniophila Murr with Radix Isatidis inhibit proliferation and metastasis of breast cancer cells. J Chin Med Assoc 81:520-530

Lowe AJ, Jourde B, Breyne P, Colpaert N, Navarro C, Wilson J, Cavers S (2003) Fine-scale genetic structure and gene flow within Costa Rican populations of mahogany (Swietenia macrophylla). Heredity 90(3):268-275

Meng F, Xing G, Li Y, Song J, Wang Y, Meng Q, Lu J, Zhou Y, Liu Y, Wang D (2016) The optimization of Marasmius androsaceus submerged fermentation conditions in five-liter fermentor. Saudi J Biol Sci 23:S99-S105

Ming SS, Xiao MX, Hong DL (2010) Optimization of bi-direction solid fermentation for toxicity-reducing and efficacy-maintaining action of fungal fermentative products of Tripterygium wilfordii. Mycosystema 29(2):294-299

Morales RR, Agrapart V, Mencacci C, Moretti C, Frajese G, Frajese GV (2008) Functional re-differentiation of prostate cancer derived cell lines by the anti-tumoral drug mycophenolic acid (MPA). EJC Suppl 6(9):146-146

Mudge DW, Atcheson BA, Taylor PJ, Pillans PI, Johnson DW (2004) Severe toxicity associated with a markedly elevated mycophenolic acid free fraction in a renal transplant recipient. Ther Drug Monit 26(4):453-455

Nakajima Y, Sato Y, Konishi T (2007) Antioxidant small phenolic ingredients in Inonotus obliquus (persoon) Pilat (Chaga). Chem Pharm Bull 55(8):1222-1226

Okour M, Jacobson PA, Ahmed MA, Israni AK, Brundage RC (2018) Mycophenolic acid and its metabolites in kidney transplant recipients: a semimechanistic enterohepatic circulation model to improve estimating exposure. J Clin Pharmacol 58(S2):628-639 
Regueira TB, Kildegaard KR, Hansen BG, Mortensen UH, Hertweck C, Nielsen J (2011) Molecular basis for mycophenolic acid biosynthesis in Penicillium brevicompactum. Appl Environ Microbiol 77(9): 3035-3043

Shaligram NS, Singh SK, Singhal RS, Szakacs G, Pandey A (2008) Compactin production in solid-state fermentation using orthogonal array method by P. brevicompactum. Biochem Eng J 41(3):295-300

Shih IL, Lin CY, Wu JY, Hsieh C (2009) Production of antifungal lipopeptide from Bacillus subtilis in submerged fermentation using shake flask and fermentor. Korean J Chem Eng 26(6):1652-1661

Shin KO, Jeon JR, Lee JS (2006) Lactic acid fermentation of Chinese yam (Dioscorea batatasDecne) flour and its pharmacological effect on gastrointestinal function in rat model. Biotech Bioproc Eng 11(3):240-244

Sun YP, Zhu LL, Liu JS (2018) Limonoids and triterpenoid from fruit of Swietenia macrophylla. Fitoterapia 125:141-146

Vinci MC, Go E, Tarnawsky SP, Shelley WC, Banno K, Lin Y, Gil C-H, Blue EK, Haneline LS, O’Neil KM, Yoder MC (2018) Mycophenolic acid induces senescence of vascular precursor cells. PLoS One 13(3):e0193749

Vinokurova NG, Ivanushkina NE, Kochkina GA, Arinbasarov MU, Ozerskaya SM (2005) Production of mycophenolic acid by fungi of the genus Penicillium link. Prikl Biokhim Mikrobiol 41(1):95-98

Wang Y, Yao Z, Liang W, Yang D, Wang W, Teng L (2016) 9 Studies on the optimization of submerged fermentation medium and conditions for Tricholoma matsutake. J Investig Med 64:A3-A4

Wold CW, Kjeldsen C, Corthay A, Rise F, Christensen BE, Duus JØ, Inngjerdingen KT (2018) Structural characterization of bioactive heteropolysaccharides from the medicinal fungus Inonotus obliquus (Chaga). Carbohydr Polym 185:27-40

Xia TU, Pan Y (2010) The bi-directional fermentation technology, a new approach to attenuating the toxicity of toxic Chinese Materia Medica. J Fungal Res 1:52-56

Xu ZN, Yang ST (2007) Production of mycophenolic acid by Penicillium brevicompactum immobilized in a rotating fibrous-bed bioreactor. Enzym Microb Technol 40(4):623-628

Xu X, Zhang X, Nong X, Jie W, Qi S (2017) Brevianamides and mycophenolic acid derivatives from the deep-sea-derived fungus Penicillium brevicompactum DFFSCS025. Mar Drugs 15(2):43. https://doi.org/10.3390/md15020043

Yang Y, Du XJ, Li P (2014) An optimized method for the preparation of Monascus purpureus DNA for genome sequencing. Appl Mech Mater 563(6):379-383

Yi Z, Xie XM, Zhang LY (2007) The origin, development and its advantage and potential of "the bi-directional solid fermentation" for medicinal fungi. Edible Fungi China 2:1-6

Zhang Q, Yang B, Li F, Liu M, Lin S, Wang J, Xue Y, Zhu H, Sun W, Hu Z, Zhang Y (2018) Mycophenolic acid derivatives with immunosuppressive activity from the coral-derived fungus Penicillium bialowiezense. Marine Drugs 16(7):230. https://doi.org/10.3390/ md16070230

Publisher's note Springer Nature remains neutral with regard to jurisdictional claims in published maps and institutional affiliations. 